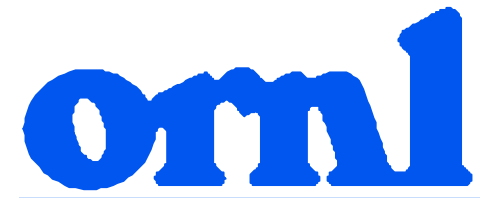

OAK RIDGE NATIONAL LABORATORY

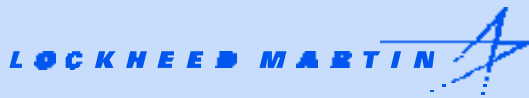

MANAGED AND OPEFATED FY

LOCKHEED MARTIN ENEFGY FESEAFGH COFPOFATION FOF: THE UNITED STATES DEPARTMENT OF ENEFGY
SNS Doc. No. 102020000TR0001R00

ORNL/TM-1999/206

\title{
Spallation Neutron Source Availability Top-Down Apportionment Using Characteristic Factors And Expert Opinion
}

Jack C. Schryver

M. Jonathan Haire 
This report has been reproduced directly from the best available copy.

Available to DOE and DOE contractors from the Office of Scientific and Technical Information, P.O. Box 62, Oak Ridge, TN 37831; prices available from (615) 576-8401.

Available to the public from the National Technical Information Service, U.S. Department of Commerce, 5285 Port Royal Rd., Springfield, VA 22161.

This report was prepared as an account of work sponsored by an agency of the United States Government. Neither the United States nor any agency thereof, nor any of their employees, makes any warranty, express or implied, or assumes any legal liability or responsibility for the accuracy, completeness, or usefulness of any information, apparatus, product, or process disclosed, or represents that its use would not infringe privately owned rights. Reference herein to any specific commercial product, process, or service by trade name, trademark, manufacturer, or otherwise, does not necessarily constitute or imply its endorsement, recommendation, or favoring by the United States Government or any agency thereof. The views and opinions of authors expressed herein do not necessarily state or reflect those of the United States Government or any agency thereof. 
SNS Document Number: 102020000TR0001R00

ORNL/TM-1999/206

\title{
SPALLATION NEUTRON SOURCE AVAILABILITY TOP-DOWN APPORTIONMENT USING CHARACTERISTIC FACTORS AND EXPERT OPINION
}

\author{
Jack C. Schryver \\ Computer Science and Mathematics Division \\ Oak Ridge National Laboratory \\ M. Jonathan Haire \\ Spallation Neutron Source Project \\ Chemical Technology Division \\ Oak Ridge National Laboratory \\ Date Published: October 1999 \\ Prepared by \\ OAK RIDGE NATIONAL LABORATORY \\ Oak Ridge, Tennessee 37831-6285 \\ Prepared for the \\ U.S. Department of Energy \\ Office of Science \\ LOCKHEED MARTIN ENERGY CORPORATION \\ managing the \\ Spallation Neutron Source Activities at the \\ Argonne National Laboratory \\ Brookhaven National Laboratory Lawrence Berkeley National Laboratory \\ Los Alamos National Laboratory Oak Ridge National Laboratory \\ under contract DE-AC-05-96OR22464 \\ for the \\ U.S. DEPARTMENT OF ENERGY
}




\section{CONTENTS}

$\begin{aligned} 2 & \text { Page }\end{aligned}$

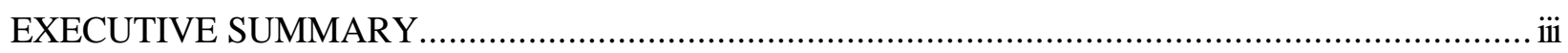

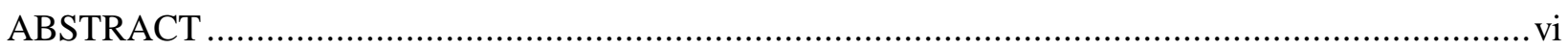

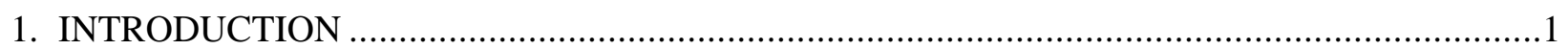

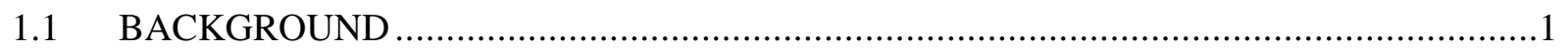

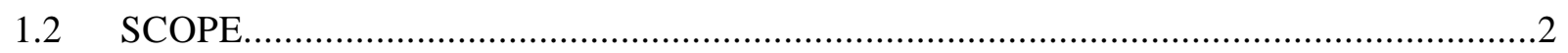

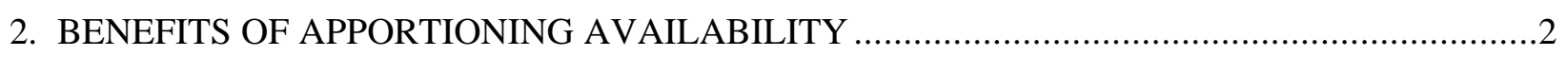

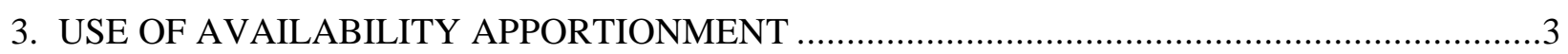

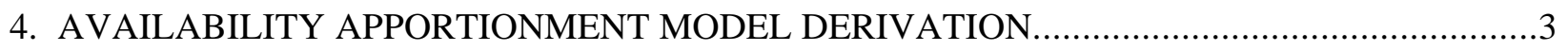

5. EXPERT SELECTION, BIAS, ELICITATION AND AGGREGATION ........................................

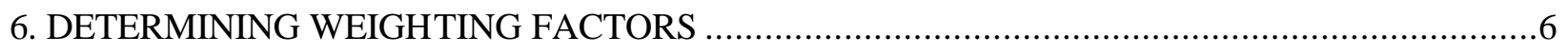

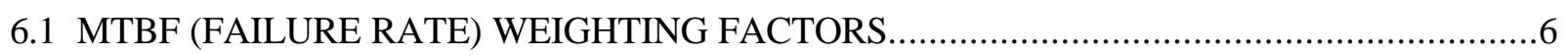

6.2 MTTR WEIGHTING FACTORS …………………….................................................

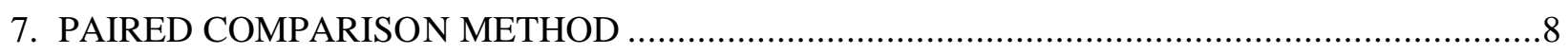

8. APPLICATION TO THE SPALLATION NEUTRON SOURCE (SNS) PROJECT …....................

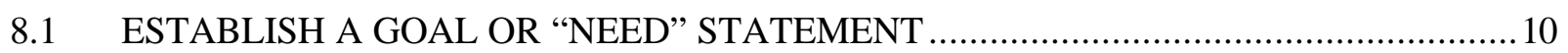

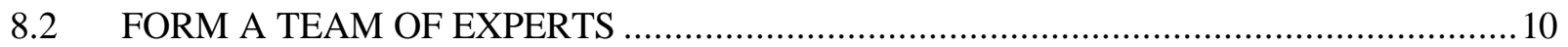

8.3 DECIDE UPON A METHODOLOGY ……………................................................ 10

8.4 AGREEMENT ON WEIGHTING FACTOR CRITERIA.................................................. 11

8.5 EXPERTS PROVIDE SCORES FOR WEIGHTING FACTORS ......................................11

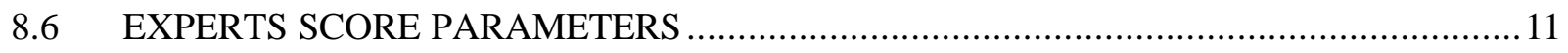

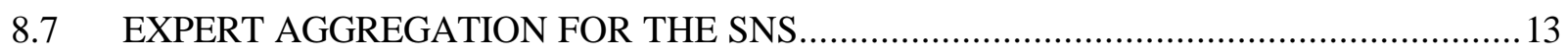

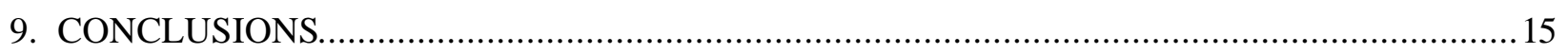

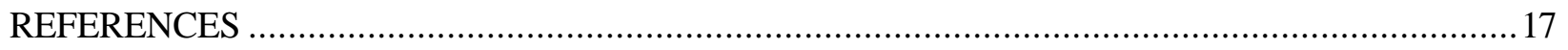




\section{EXECUTIVE SUMMARY}

\section{Introduction}

System design engineers must translate permitted overall facility downtime into detailed design and operating specifications for numerous systems and subsystems that make up a facility. The process of assigning reliability and maintainability requirements to individual equipment systems to attain their desired overall availability is known as availability apportionment. Apportionment is normally required early in a program when little or no hardware information is available. Apportionment is a continuous process during design and development, with subsequent re-allocations performed as further information regarding the contribution of various subsystem elements to the top-level requirement becomes available. Apportionment, when coupled with availability prediction, enables the selection of viable alternative configurations, identifies problem areas, and provides redirection of the program into more productive areas as necessary. A method for apportioning overall facility availability requirements among systems and subsystems is presented. The application of this methodology to the Spallation Neutron Source (SNS) facility is given as an example of apportionment.

Typically, availability criteria for individual equipment systems is established using a bottom-up approach. This approach combines reliability and maintainability performance requirements for individual equipment units to predict overall facility availability. The calculated value is then compared to the design requirement, and through an iteration process, input information to the computation is adjusted, and the calculations are repeated until they match the facility availability goal. This method requires a detailed design.

A top-down approach is intuitively more satisfying. This method takes an overall facility requirement and divides it among facility systems first, then among subsystems. The overall facility availability requirement is distributed proportionately among the facility systems, based on each systems capability for meeting a design objective. The approach imposes higher availability requirements on those systems in which an incremental increase in availability is easier to achieve, and lower availability requirements where an increase is more difficult and costly. An optimization such as this results in lower facility costs.

\section{Determining Weighting Factors}

Characteristics that influence equipment reliability and maintainability are described. Experts, using engineering judgment, score each characteristic for each system whose availability design goal is to be established. The Analytic Hierarchy Process method is used to produce a set of weighted rankings for each characteristic of each alternative system. Expert elicitation relies on a series of exhaustive pairwise comparison judgments.

Characteristics chosen that influence reliability are system complexity, design immaturity, and a stressful operating environment. System complexity may be evaluated by considering the probable number of parts or components that make up the system - the higher the number of parts the more likely there will be failures. The lack of a proven design increases the probable number of failures. Components operating in stressful conditions, e.g. high temperatures, vibration, radiation fields, have more failures than those operating in benign environments. 
Characteristics that influence maintenance are the additive elements of repair time: fault detection and diagnosis time, preparation time to conduct the repair, fault correction time itself, and time to restart the system once the fault is corrected.

\section{The Paired Comparison Method}

The Analytic Hierarchy Process method is used to produce sets of weighting factors for each alternative. The paired comparison procedure is implemented in two phases. During the first phase, the relative importances of "characteristics" are established. Experts are asked, for example, to compare complexity and design maturity with respect to their importance in apportioning reliability. The pairs (complexity and design immaturity in this example) are compared, i.e. given a score, using a scale from 1 to 9. The second phase compares pairs of systems for each characteristic. For example, for the SNS, experts would be asked to compare the complexity of the ring and linac systems. All pairs are evaluated for each characteristic. For SNS six systems (subprojects) were compared for each of the three characteristics of reliability. The entire procedure is repeated twice, first for reliability and then for maintainability. Availability is allocated based on a joint consideration of reliability and maintainability. The Expert Choice $^{\mathrm{TM}}$ software package was used to assist experts in working through the Analytic Hierarchy Process method.

A principal technical expert was chosen to represent each subproject. A structured interview was conducted for each expert individually. Team aggregation and final adjustments of availability allocations were performed at the conclusion of structured interviews, and results were reviewed.

\section{Application to the Spallation Neutron Source (SNS) Project}

The SNS is a new accelerator-based neutron scattering facility which will provide special scientific and research capabilities to universities, industry, private laboratories, federal laboratories and others involved in the development and application of neutron-based research. The SNS is a collaborative effort among five national laboratories for design, construction, installation, and commissioning. The SNS Project is divided into subprojects according to the responsibilities of each national laboratory. Clearly, with responsibilities widely distributed around the country, it is essential that the $90 \%$ overall facility inherent availability requirement be apportioned among subprojects in order to have a clearly defined availability design criteria for each major system. Design and construction of the SNS at Oak Ridge, Tennessee are expected to be completed in the year 2005 and cost approximately $\$ 1.3$ billion.

\section{Results}

This report presents the recommended apportioned availability for each major system or subproject of the SNS facility. There are many experiment instrument stations in the SNS, and experiments are conducted whenever neutrons are produced (i.e. the experiment systems subproject is assigned a $100 \%$ availability requirement.) The control, conventional facility, and ion source systems were given high scores by experts. Thus, they have extremely high availability requirements. On the other hand, target systems were awarded low scores and have the lowest aggregate availability requirement.

Correlation analyses demonstrated a high consistency in inter-expert weighted rankings of SNS subprograms. The range of correlations among expert pairs was from 0.685 to 0.985 , with $73 \%$ of the correlations above 0.800 . The correlations of expert's weighted ranks with the aggregate scores ranged 
from 0.742 to 0.988 . We concluded that the aggregate apportioned availability among subprojects represents each individual expert's responses very well.

The agreement of the apportioned availability with availability data from existing accelerator facilities varied widely, reinforcing the conclusion that previous data from similar facilities cannot be blindly used to apportion availability for a new, one-of-a-kind facility. The AGS availability scores agreed very well with the aggregate scores for the SNS (.96); the agreement with ISIS was moderate (.345); however, the correlation with LANSCE H+ was actually negative (-.292)!

Availability scores for each expert were submitted to a principal components analysis in order to represent expert judgment in a lower dimensional space. Expert's scores are plotted with respect to the first two principal components. Three natural clusters emerged. The largest cluster consisted of experts representing the ring, target, and conventional facilities. The aggregate scores were also located in this cluster. A smaller cluster contained experts for the Linac and source, and a one-member cluster represented the source. These two smaller clusters differed from the main cluster in their estimates of higher relative target availability. The singleton outlier cluster also differed from other experts by allocating a lower control systems availability. 


\begin{abstract}
Apportionment is the assignment of top-level requirements to lower tier elements of the overall facility. A method for apportioning overall facility availability requirements among systems and subsystems is presented. Characteristics that influence equipment reliability and maintainability are discussed. Experts, using engineering judgement, scored each characteristic for each system whose availability design goal is to be established. The Analytic Hierarchy Process (AHP) method is used to produce a set of weighted rankings for each characteristic for each alternative system. A mathematical model is derived which incorporates these weighting factors. The method imposes higher availability requirements on those systems in which an incremental increase in availability is easier to achieve, and lower availability requirements where greater availability is more difficult and costly. An example is given of applying this top-down apportionment methodology to the Spallation Neutron Source (SNS) facility.
\end{abstract}




\section{INTRODUCTION}

Facilities achieve their goals by operating at less than $100 \%$ availability. Systems design engineers must translate permitted overall facility downtime into detailed design and operating specifications for the numerous systems and subsystems that make up a facility. The process of assigning reliability and maintainability requirements to individual equipment systems to obtain their desired overall availability is known as availability apportionment (or budgeting). An overall facility availability goal or requirement is allocated among facility systems and subsystems.

Typically, availability criteria for individual equipment systems are established using a bottom-up approach. ${ }^{1}$ This approach combines reliability and maintainability performance requirements for individual equipment units to predict an overall facility availability. The calculated value is then compared to the design requirement, and through an iteration process, input information to the computation is adjusted, and the calculations are repeated until they match the facility availability goal. The input data used in the final iteration become the system and subsystem availability design criteria. A typical application of the bottomup approach is electronic system design. The bottom-up approach requires a well-defined, detailed design and equipment performance data. The lack of this information prevents the application of the bottom-up approach to novel technologies or in conceptual, early facility design phases.

A top-down approach is intuitively more satisfying. This method takes an overall facility requirement and divides it among facility systems first, then among subsystems. However, more is involved than a simple mathematical equality. For example, the availability of an individual system varies with the complexity of the unit, its design maturity, its operating environment, and the accessibility to the failed unit for repair. These are just a few of the more important factors. An overall facility availability requirement should be distributed proportionately among the facility systems, based on each systems capability for meeting a design objective. An optimization such as this results in lower facility costs. This paper expands one of its author's (Haire) earlier works to include recent knowledge. These findings will be applied to the Spallation Neutron Source (SNS) as an example.

\subsection{BACKGROUND}

Top-down methods of allocating availability have been used for some time. In 1957, the AGREE allocation method ${ }^{2}$ was developed for reliability apportionment. The method was based on unit or subsystem complexity rather than failure rates. In 1965 the Karmiol method ${ }^{3}$ utilized the following factors of influence to apportion overall reliability: complexity, state-of-the-art, operational profile, and criticality of the system to mission objectives. More recently, Snedley ${ }^{4}$ employed a similar hierarchical technique called Feasibility-of-Objective to allocate reliability among LEB ring magnet power systems in the superconducting super collider. The Karmiol method provides for equal weighting of all factors. Equal factor weighting breaks down when some factors are more important than others in apportionment.

The Bracha method $^{5}$ (introduced in 1964) allocated reliability using four factors: state-of-the-art, subsystem complexity as estimated by number of parts, environmental conditions, and relative operating time. In 1985, Haire, Maltese, and Sohmer ${ }^{6}$ combined factors of influence for reliability and maintainability to allocated facility availability. In 1998, Virtanen ${ }^{7}$ used the Analytical Hierarchy Process (AHP) ${ }^{8}$ to determine the complexity factor in allocating dependability requirements. 
Virtanen's research ${ }^{7}$ is an important advancement and is relevant to the top-down approach. However, this work has some weaknesses. Virtanen describes allocation of reliability, availability, and maintainability individually. Availability should be allocated based on joint consideration of reliability and maintainability. The psychology of expert elicitation suggests that questions be asked in the simplest, most unambiguous terms. Virtanen used undefined functions in allocating availability; the IEC Standard 706-6 was used in allocating maintainability. Virtanen's greatest contribution was the use of the AHP to estimate the complexity influence factor in allocating reliability

AHP was first described in 1980 by Thomas Saaty ${ }^{9}$ and has since been used in numerous applications as a decision-making framework that organizes the dimensions of a complex system into a hierarchic structure. It provides a systematic procedure to decompose a problem into smaller constituent parts. The basic expert elicitation process relies on a series of exhaustive pairwise comparison judgments. Pairwise judgments depend on relative comparison, in contrast to absolute comparison. In absolute comparison, alternatives are matched with an abstract internal standard that has been developed through experience. However, relative comparison occurs with actual pairs of alternatives according to a common attribute(s). Relative comparisons are cognitively easier and more basic than absolute comparisons.

\subsection{SCOPE}

This work updates Haire's 1985 work by using AHP to compute influence weighting factors for reliability and maintainability that are then used to calculate a facility's systems availability from an overall availability requirement. The AHP is a mature technology that has been broadly applied in many different fields, including (to name a few): software reliability apportionment ${ }^{10}$, conflict resolution ${ }^{11}$, financial cutback decision-making $^{12}$, performance evaluation ${ }^{13}$, layout design ${ }^{14}$, medical decision-making ${ }^{15}$, traffic planning $^{16}$, energy policy planning ${ }^{17}$, facility site selection ${ }^{18}$, ship design ${ }^{19}$, neural impulse firing modeling $^{20}$, and risk analysis. ${ }^{21}$ The method has also been used in the field of reliability engineering, as seen in Virtanen's work. It provides a very generic, formal and traceable method for expert elicitation and aggregation in the context of multiattribute decision-making. Several software products are available to support AHP calculations, including Expert Choice ${ }^{\mathrm{TM}}$, and a module in SAS to calculate AHPs.

\section{BENEFITS OF APPORTIONING AVAILABILITY}

Availability apportionment has the following benefits ${ }^{1}$ :

1. Designing to an availability requirement, rather than the well-meaning, "we will do the best that we can" approach, quantifies system availability requirements and forces subprojects (subcontractors) to consider availability equally with other systems parameters.

2. Through subproject comparisons between system availability requirements and predictions, weak links or critical components that prevent achieving an availability requirement can be identified. Efforts to resolve these weak links should improve the state-of-the-art of all facets of facility design.

3. Availability enhances design integration by focusing attention on the relationship between facility, system, subsystem, and component reliability and maintainability. 
4. Requirements determined through an apportionment procedure will be more realistic, consistent, and economically attainable than those obtained through subjective methods or those resulting from retrofits from field experiences.

5. Apportionment should result in optimum facility availability since the allocating method considers those factors that influence availability such as complexity, design maturity, operating environment, repair times, etc.

\section{USE OF AVAILABILITY APPORTIONMENT}

Apportionment is required whenever a facility owner must decide how available various systems and subsystems must be in order to design a product that will meet a specified availability requirement ${ }^{1}$. Apportionment is normally required early in the program when little or no hardware information is available. Apportionment is a continuous process during design and development, with subsequent reallocation interactions performed as further information regarding the contribution of various subsystem elements to the top-level requirement becomes available. Apportionment, when coupled with availability prediction, enables the selection of viable alternative configurations, identifies problem areas, and provides redirection of the program into more productive areas as necessary. Frequently, apportionment and tradeoff studies go together so that the overall goal is apportioned in a manner that will optimize some other important parameter such as cost.

Project apportionment and re-apportionment should be considered:

- Initially, in the early conceptual design stage.

- At the conclusion of major design phases.

- At the initiation of any major design revision.

- At the conclusion of major testing phases.

- At the conclusion of qualification or acceptance testing for the majority of functional systems and components.

\section{AVAILABILITY APPORTIONMENT MODEL DERIVATION}

Consider a system consisting of $n$ subsystems, all of which are required to perform the overall system's function. The availability of the system is 


$$
A_{s}=\frac{\boldsymbol{\theta}_{s}}{\Theta_{s}+R_{s}}
$$

where

$\mathrm{A}_{s}=$ system inherent, or intrinsic, availability goal;

$\theta_{\mathrm{s}}=$ system Mean Time Between Failure (MTBF); and

$R_{s}=$ system Mean Time to Repair (MTTR).

The basic formula relating the availability of $n$ subsystems (i.e., the failure of any one will shut the system down) is

$$
A_{s}=\prod_{i=1}^{n} A_{i}
$$

Expressing Equation 2 in terms of subsystems MTBF, $\theta_{i}$, and MTTR, $R_{i}$, and rearranging gives

$$
\frac{1}{A_{s}}=1+\frac{R_{s}}{\boldsymbol{\Theta}_{s}}=\prod_{i=1}^{n} \frac{1}{A_{i}}
$$

Apportioning the system availability requirement to the subsystem level requires a weighting factor that reflects the relative functional importance of a subsystem to system performance as well as accounting for factors that affect the availability of the subsystem. This weighting factor must exhibit two main properties, namely:

- Its magnitude should increase as the availability of the subsystem increases.

- Its formulation must satisfy the bounds of $\mathrm{A}_{s}$ and each $\mathrm{A}_{i}$.

Let $z_{i}$ be the failure rate weighting factor for the $i^{\text {th }}$ subsystem where $\Sigma \mathrm{z}_{\mathrm{i}}=1$. The MTBF allocated to each subsystem is defined by:

$$
\boldsymbol{\Theta}_{i}=\frac{\sum z_{i}}{z_{i}}\left(\boldsymbol{\theta}_{s}\right)=\frac{\boldsymbol{\theta}_{s}}{z_{i}}
$$

The failure rate $\lambda_{i}$ is the reciprocal of the MTBF such that

$$
\lambda_{i}=\frac{1}{\theta_{i}}=\frac{z_{i}}{\theta_{s}}
$$

Let $\mathrm{y}_{i}$ be the MTTR weighting factor for the $i^{\text {th }}$ subsystem where $\Sigma \mathrm{y}_{\mathrm{i}}=1$. The MTTR allocated to each subsystem is weighted by the frequency of failure and is defined by

$$
R_{i}=y_{i}\left|\frac{\sum_{i=1}^{n} \lambda_{i}}{\mid \sum_{i=1}^{n} \lambda_{i} y_{i}}\right|^{R_{s}}
$$


Substituting Eq. 5 into Eq. 6, and dividing the modified Equation 6 by Equation 4 yields

$$
\frac{R_{i}}{\boldsymbol{\Theta}_{i}}=\left\{\frac{y_{i} z_{i}}{\sum_{i=1}^{n} y_{i} z_{i}}\right\rceil\left(\frac{1}{A_{s}}-1\right)
$$

Equation 7 establishes the $\mathrm{R}_{\mathrm{i}} / \theta_{i}$ ratio for the allocated equipment availability in terms of system availability and the MTBF and MTTR weighting factors $z_{i}$ and $y_{i}$, respectively.

This final Equation does not consider such parameters as duty cycle and functional importance. The failure of any subsystem is assumed to shut the system down. In this simple treatment, there is no hierarchical functional importance such as safety systems versus process systems. A more detailed derivation that considers these parameters is given in Haire's 1985 work.

Since

$$
\frac{1}{A_{i}}=\frac{1}{1+\left(R_{i} / \theta_{i}\right)}
$$

Equation 7 explicitly relates the allocated availability of the $i^{\text {th }}$ system, $\mathrm{A}_{i}$, to the overall system availability goal, $\mathrm{A}_{s}$. Qualitative characteristics that influence reliability and maintainability weighting factors, are present in the MTTR, $R_{i}$, and MTBF, $\theta_{i}$ ratio in Equation 7.

\section{EXPERT SELECTION, BIAS, ELICITATION AND AGGREGATION}

The weighting factors in Equation 7 are determined from the quantitative analysis of expert judgments. Methods for expert judgment have been studied extensively. ${ }^{22}$ Some that are pertinent to availability apportionment are expert selection, bias, elic itation, calibration and aggregation.

Identification of experts and sample size is an important issue in availability apportionment for which there exists little guidance. ${ }^{22}$ For a large complex facility composed of several major subprograms (e.g., the SNS) the sample should fairly represent each major subproject and technology area. Subject matter experts are drawn from the design team because they have the most in-depth knowledge and are major stakeholders in the facility design. The area of knowledge and subproject with which the expert is associated influences expert bias. Thus, the effect of expertise is complex because it should increase both accuracy in terms of the internal judgment and response bias. One method of evenly distributing bias is to ensure that each subprogram is equally represented in a stratified sample. 
At the individual level, the AHP method is a detailed unambiguous procedure for expert elicitation based on paired comparison judgments. Van Steen ${ }^{22}$ outlines the group dynamics of expert elicitation for several alternative strategies. Teams can be structured to meet separately or jointly. They can be charged to obtain a consensus judgment or retain individual judgments that are subsequently aggregated. Hybrid strategies may also be efficient in certain instances where experts are geographically distributed, and have numerous demands upon their time. Initially individuals composing the expert sample can be interviewed separately. Subsequently the analyst can identify differences of judgment. During the second phase, the expert sample meets jointly, and issues and conflicts are discussed. Some issues will be resolved because of misunderstanding or deficiencies of knowledge in certain areas, and other issues may remain after discussion due to fundamental differences in opinion or perspective. A final interview can be conducted for each expert after the joint discussion in order to allow the expert to incorporate their enhanced understanding into their judgments, or the group may strive to achieve a consensus judgment. The latter is desirable because the experts will "own" the result, and will likely support the findings. If experts become mired in details of the elicitation procedure, they may be inclined to distance themselves from the outcome.

A wide range of aggregation techniques has been used in quantitative analysis of expert judgment. The AHP process as implemented in Expert Choice $^{\mathrm{TM}}$ uses an averaging technique. The weighted averaging aggregation technique calculates the weighted rank of each aggregated alternative $\mathrm{W}_{\mathrm{j}}$ as:

$$
W_{j}=\sum_{i=1}^{m} \alpha_{i} W_{i j}
$$

where $\mathrm{w}_{\mathrm{i} 1}, \ldots, \mathrm{w}_{\mathrm{im}}$ are the weighted rankings generated by the $i$ th expert, and $\alpha_{\mathrm{i}}$ is the weighted ranking of the $i$ th expert. The $\alpha_{I}$ 's sum to unity. Typically experts are equally weighted; however in light of detailed or objective and quantitative information about the expert's knowledge and bias, unequal weights

are sometimes assigned to expert samples. For example, Zio ${ }^{23}$ outlines a formal process utilizing AHP to assign weighted rankings to experts based on personal knowledge, sources of information, unbiasedness, relative independence, personal interest, past experience, and a performance measure.

\section{DETERMINING WEIGHTING FACTORS}

Weighting factors are determined by surveying experts for their opinions regarding the design and operation of facility equipment. Experts are questioned about characteristics that affect MTBF and MTTR. The following paragraphs provide typical characteristics that influence these key parameters.

\subsection{MTBF (FAILURE RATE) WEIGHTING FACTORS}

Failure rate was selected as the operational definition of reliability because it is a more intuitive concept than MTBF. A greater failure rate simply means more failure. Each factor is scored on a bi-directional nine-point scale in the paired comparisons. A score of nine indicates the greatest difference between alternative subprograms, and a score of one indicates equality between alternatives with respect to the factor under consideration. The factors considered are: 
- System Complexity. The complexity of a system will have an effect on the achievable reliability. As the number of subsystems and components increase, the more difficult and costly it becomes to achieve high system MTBF (and a low failure rate). Imposing an unrealistically high MTBF on the more complex systems increases cost disproportionately when compared with the alternative of increasing MTBF requirements for simpler systems. Subsystem complexity may be evaluated by considering the probable number of parts or components making up the system.

- Design Immaturity. The amount of research and development required to produce the systems will greatly influence the cost and schedule of development. Imposition of a high MTBF requirement on a system under development will increase the development time, the number of tests required to obtain the MTBF, and the costs. Equipment considered present state-of-the-art is penalized less by high MTBF requirements. The state of the present engineering progress in all fields is considered.

- Operation Time. The fraction of system operating time and the length of time that the system is required to perform influences the achievable MTBF value. More development effort and cost will be required to produce a system capable of operating full-time for long periods without failure than to develop one for shorter periods of use or for infrequent, intermittent operation.

- Stressful Environmental Conditions. The intended operational environment will influence the achievable MTBF value. A system operating at extremes of temperature, pressure, mechanical, corrosion and erosion stresses will tend to cost more to develop than a similar system used under less severe conditions. Systems expected to experience harsh and extreme conditions during performance will have a higher failure rate.

The above characteristics are not unique or all-inclusive. Parameters influencing failures should be tailored to the application.

\subsection{MTTR WEIGHTING FACTORS}

A high weighted ranking is given to the system if the time element described below is long, and a low score is given if it is short. The factors considered are:

- Fault Detection. This element of time includes verification that a malfunction exists and determination of its character by such means as displays, instruments, facility operating data, inspection, special tests, etc.

- Fault Diagnosis. Considerations used in evaluating this time element include the visibility and nature of the fault in the system where it may occur. This may range from visual checks to interpretations of the symptoms via considerable knowledge and experience, special test procedures, review of extensive data and records by specialized engineers, etc.

- Preparation Time. This factor addresses the time between the diagnosis of the failure and the start of the maintenance action. It includes obtaining permission to conduct the repair, obtaining the spare part, formation of the maintenance team, depressurization, cool-down or warm-up, switching power, preparation of rigging, establishment of a safe maintenance environment, etc. 
- Fault Correction. This activity is made up of disassembly, installation, adjustment, assembly and calibration times. The time required depends on the nature of the system, plug-in parts, modular parts requiring standard tools, removal of assemblies, special tools and techniques, difficult alignment, weldments, heat treatment, cleanup, etc.

- Checkout Time. Considerations involving checkout of a system after repair include need for special equipment, involvement of other systems, automated checkout operations, personnel evacuation prior to checkout, etc.

As for MTBF characteristics, factors influencing MTTR are not unique or all-inclusive. Factors should be developed and tailored to specific applications.

\section{PAIRED COMPARISON METHOD}

The AHP method is used to produce a set of weighted ranks, $w_{i}$, for each alternative, subject to the constraint that $\Sigma w_{\mathrm{i}}=1$. Simple hierarchies were developed for reliability under the goal of apportioning failure rate among systems and maintainability under the goal of apportioning MTTR among systems. The criteria (i.e., weighting factors such as complexity, immaturity, and stress environment for reliability) comprise the first level under the goal statement, and every subproject alternative (e.g., source, linac, ring, target) is listed under each criterion. The weighted ranking $w_{\mathrm{i}}$ for each alternative is computed using a simple additive model:

$$
w_{i}=\sum_{i=1}^{m} c_{i} v_{i j}
$$

where $c_{1}, c_{2}, \ldots c_{m}$ are the weighted ranks of the criteria, $i$, themselves $\left(\sum c_{i}=1\right)$, and $v_{i j}$ are the weighted rankings of the alternatives, $j$, under each criterion. The paired comparison procedure is implemented in two phases. During the first phase, criteria are ranked with respect to importance toward the overall goal (reliability or maintainability). The entire procedure is repeated twice, first for reliability and then for maintainability. All $\mathrm{n}(\mathrm{n}-1)$ pairs are compared using a scale from 1 to 9 , where 1 ndicates equal importance and 9 is the most extreme level of importance of the top over the bottom alternative. By inverting the top alternative, another 9 levels of importance are available to compare the degree of importance of the new alternative. The experts would be asked, for example, to compare complexity and design maturity with respect to their importance in apportioning reliability. The weighted rankings of the criteria $\mathrm{c}_{\mathrm{i}}$ are determined using the matrix procedure. ${ }^{8}$

After completing the weighted ranking of criteria, each subproject alternative pair is evaluated with respect to the first criterion. When all pairs have been compared, the next criterion is selected and each pair is compared again. The paired comparison process is repeated for every criterion. Expert Choice ${ }^{\mathrm{TM}}$ was installed on a laptop computer to assist the expert in working through the AHP method. The program provides several comparison formats; we discovered that the questionnaire format was most intuitive for our expert sample (see Section 8.5).

We discovered that working purely with paired comparison judgments was difficult for our expert sample. When providing responses to the first few comparisons, experts attempted to reference an approximate 
internal ranking of alternatives to help instantiate or anchor the comparison process. Accordingly, we encouraged experts to generate a rough ranking of alternatives before proceeding with the paired comparisons. After completing several judgments, the mental ranking might undergo some refinement. Some experts also preferred to initially locate the most extreme alternatives, and anchor the paired comparison process by completing those first.

It is important to maintain mathematical consistency in wording questions posed to experts when administering the paired comparison procedure. For example, the reliability goal is to "allocate failure rate among systems." If the goal is simply to allocate reliability, the subject matter expert may interpret reliability as either MTBF or failure rate - however, the two terms are inversely related. Another critical consideration is to ensure that all criteria statements are correctly aligned with each other and the goal statement. Thus, complexity is a more appropriate criterion than its antonym simplicity because greater complexity increases failure rate. Likewise, immaturity aligns with failure rate instead of maturity.

The matrix of pairwise comparisons is the source for the principal eigenvector that is equivalent to the vector of weighted rankings. After normalization, the maximum eigenvalue, $\beta_{\max }$, is a measure of consistency, or the degree to which the transitivity relation is satisfied in the matrix of paired comparisons. Transitivity among three elements is preserved when all distances among the elements can be represented on a single underlying dimension. The elements $\mathrm{A}, \mathrm{B}$ and $\mathrm{C}$ are transitive if and only if $\mathrm{A}>\mathrm{B}$ and $\mathrm{B}>\mathrm{C}$ implies that $\mathrm{A}>\mathrm{C}$. A normalized consistency ratio $0<\mathrm{CR}<1$ is given by:

$$
C R=\left[\left(\beta_{\max }-n\right) /(n-1)\right] / R I
$$

where $\mathrm{n}$ is the order of the matrix and random index (RI) is the statistical mean of randomly generated matrices of the same order.

After completing a matrix of paired comparisons, the expert is allowed to view the weighted ranking and a consistency index. If the observed ranking seems counterintuitive, or the consistency index is too high (> 0.1 ), the expert is allowed to return to the previous page to revise their judgments. The judgments from an $\mathrm{n} \times \mathrm{n}$ matrix are consistent if and only if:

$$
\mathrm{a}_{\mathrm{ij}} \mathrm{a}_{\mathrm{jk}}=\mathrm{a}_{\mathrm{ik}} \quad(\mathrm{i}, \mathrm{j}, \mathrm{k}=1, \ldots, \mathrm{n})
$$

In other words, the judgments must satisfy strong transitivity conditions on a ratio scale. Roughly speaking, the consistency index indicates the degree to which the judgments should be adjusted to restore transitivity.

\section{APPLICATION TO THE SPALLATION NEUTRON SOURCE (SNS) PROJECT}

The SNS is a new accelerator-based neutron scattering facility which will provide special scientific and research capabilities to the Nation's universities, industry, private laboratories, federal laboratories and others involved in the development and application of neutron-based research. Neutrons are unique and increasingly essential as a tool in broad areas of physical, chemical, and biological sciences, as well as in 
new materials development. The design and construction of the SNS at Oak Ridge, Tennessee are expected to be completed in the year 2005 and cost approximately $\$ 1.3$ billion.

The SNS is a collaborative effort among five national laboratories, who will design, construct, install, and commission the facility. The SNS Project is divided into subprojects according to the responsibilities of each national laboratory. Oak Ridge National Laboratory (ORNL) in Oak Ridge, Tennessee is the lead laboratory and the site where the facility will be located. ORNL will provide overall coordination and direction for the Project, and will provide the target system. Argonne National Laboratory in Illinois is responsible for experiment instrumentation. The front-end source system is the responsibility of Lawrence Berkeley National Laboratory in California. Los Alamos National Laboratory in New Mexico will provide the linear accelerator system. The ring and transfer lines system is the responsibility of Brookhaven National Laboratory in New York. Clearly, with responsibilities widely distributed around the country, it is essential that the $90 \%$ overall facility inherent availability requirement be apportioned among subprojects in order to have a clearly defined availability design criteria for each major system. The following sections represent the steps that should be conducted using availability apportionment for the SNS Project.

\subsection{ESTABLISH A GOAL OR “NEED” STATEMENT}

In general, there must be consensus among those affected that the apportioned overall availability among systems is needed and that the resulting budgeted system availabilities will be used to guide design. In this example, the carefully worded "need" statement pertaining to the SNS for availability apportionment is, "A Project office approved, senior team leaders agreed on, set of level two work breakdown structure availability allocations".

\subsection{FORM A TEAM OF EXPERTS}

The experts chosen for the SNS Project availability apportionment example were the senior team leaders and project manager. Senior team leaders are those managers who lead the design, research and development, procurement, construction, and demonstration testing at each five national laboratories. A senior team leader may designate someone to act for them, and may have consultants advise them, but there is only one vote per subproject. Thus, each technical area is represented and biases should be evenly distributed. It is these managers that must meet availability requirements. A team of six experts was selected to have a vote in the final aggregate ranking for the six subprojects.

\subsection{DECIDE UPON A METHODOLOGY}

In this example, the reliability, availability, maintainability, and inspectability (RAMI) task leader organizes, coordinates, and facilitates the availability apportionment exercise. The leader provides knowledge of the apportionment methodology and guidance in conducting the process. This work advocates applying the derivation of Equations shown in Section 4 of this paper, culminating in the applic ation of Equations 7 and 8. Note that the subproject availability, $A_{i}$, is simply a function of overall availability, $A_{s}$, and weighting factors, $\mathrm{y}_{\mathrm{i}}$ and $\mathrm{z}_{\mathrm{i}}$. The AHP method, which uses a "paired comparison" method as described in Section 7

of this paper, is suggested for use in scoring weighting factors. In this example, Expert Choice ${ }^{\mathrm{TM}}$ was used to input expert opinion scores, compute eigenvectors and perform matrix algebra, and to calculate 
weighted ranking values. A simple spreadsheet calculation was used to input the final weighted rankings for the subprojects based on reliability (failure rate) and maintainability goals, and calculate availabilities from Equations 7 and 8.

\subsection{AGREEMENT ON WEIGHTING FACTOR CRITERIA}

Commonly used factors that influence reliability and availability are given in Section 6 of this paper. However, these are not unique and weighting factors should be tailored to each particular application. For example, in this SNS application, operating time (Section 6.1) is not used, and fault detection and diagnosis time are combined. At the subproject level, factors chosen that influence MTTR for the SNS were complexity, immaturity, and environmental stress; factors chosen for MTTR were detection and diagnosis time, preparation time, correction time, and restart time.

\subsection{EXPERTS PROVIDE SCORES FOR WEIGHTING FACTORS}

Judgments were elicited independently from each expert in this example. Exhibit 1 shows an example of one expert's scoring of some factors that influence failure rate using the questionnaire mode in Expert Choice $^{\mathrm{TM}}$. The interpretation of Exhibit 1 is that the complexity factor is moderately to strongly more important than immaturity of design in the reliability of the SNS; thus, a score of +4 is given on the first line. Equipment system complexity is equally to moderately more important than the equipment operating in a stressful environment; thus, a score of +2 is given on line 2 . Immaturity of design is equally or moderately less important than a stressful environment; thus, a score of -2 is given on line 3 . Computing the eigenvector that results from these scores gives a weighted ranking for complexity equal to 0.571 , stressful environment equal to 0.281 , and immaturity equal to 0.143 . These are the relative importance of the criteria that influence reliability and that will be applied to each subproject. A similar procedure was followed to calculate weighted rankings that influence maintainability.

\section{Exhibit 1. Compare mean-time-between-failure criteria.}

ORNL DWG 99C-159

\begin{tabular}{|l|l|l|l|l|l|l|l|l|l|l|l|l|l|l|l|l|l|l|l|}
\hline 1 & COMPLXTY & 9 & 8 & 7 & 6 & 5 & 4 & 3 & 2 & 1 & 2 & 3 & 4 & 5 & 6 & 7 & 8 & 9 & IMMATURE \\
\hline 2 & COMPLXTY & 9 & 8 & 7 & 6 & 5 & 4 & 3 & 2 & 1 & 2 & 3 & 4 & 5 & 6 & 7 & 8 & 9 & STRESS-E \\
\hline 3 & IMMATURE & 9 & 8 & 7 & 6 & 5 & 4 & 3 & 2 & $\mathbf{1}$ & 2 & 3 & 4 & 5 & 6 & 7 & 8 & 9 & STRESS-E \\
\hline
\end{tabular}

1=Equal $3=$ Moderate $5=$ Strong $7=$ Very Strong $9=$ Extreme

\subsection{EXPERTS SCORE PARAMETERS}

For this example, Exhibit 2 shows a matrix of scores relating the importance of complexity in paired comparison judgments for every subproject. Exhibit 2 was so complex given the number of alternatives that $\mathbf{i}$ was confusing to experts in scoring. Experts appeared to be drawing paired comparisons scores from a crude underlying mental model of a simple ranking of alternatives. Thus, an aid was developed to assist experts in scoring their opinion. 
Exhibit 2. Compare subprojects with respect to complexity.

ORNL DWG 99C-160

\begin{tabular}{|c|l|c|c|c|c|c|c|c|c|c|c|c|c|c|c|c|c|c|r|}
\hline 1 & SOURCE & 9 & 8 & 7 & 6 & 5 & 4 & 3 & 2 & 1 & 2 & 3 & 4 & 5 & 6 & 7 & 8 & 9 & LINAC \\
\hline 2 & SOURCE & 9 & 8 & 7 & 6 & 5 & 4 & 3 & 2 & 1 & 2 & 3 & 4 & 5 & 6 & 7 & 8 & 9 & RING \\
\hline 3 & SOURCE & 9 & 8 & 7 & 6 & 5 & 4 & 3 & 2 & 1 & 2 & 3 & 4 & 5 & 6 & 7 & 8 & 9 & TARGET \\
\hline 4 & SOURCE & 9 & 8 & 7 & 6 & 5 & 4 & 3 & 2 & 1 & 2 & 3 & 4 & 5 & 6 & 7 & 8 & 9 & CONTROLS \\
\hline 5 & SOURCE & 9 & 8 & 7 & 6 & 5 & 4 & 3 & 2 & 1 & 2 & 3 & 4 & 5 & 6 & 7 & 8 & 9 & CONV FAC \\
\hline 6 & LINAC & 9 & 8 & 7 & 6 & 5 & 4 & 3 & 2 & 1 & 2 & 3 & 4 & 5 & 6 & 7 & 8 & 9 & RING \\
\hline 7 & LINAC & 9 & 8 & 7 & 6 & 5 & 4 & 3 & 2 & 1 & 2 & 3 & 4 & 5 & 6 & 7 & 8 & 9 & TARGET \\
\hline 8 & LINAC & 9 & 8 & 7 & 6 & 5 & 4 & 3 & 2 & 1 & 2 & 3 & 4 & 5 & 6 & 7 & 8 & 9 & CONTROLS \\
\hline 9 & LINAC & 9 & 8 & 7 & 6 & 5 & 4 & 3 & 2 & 1 & 2 & 3 & 4 & 5 & 6 & 7 & 8 & 9 & CONV FAC \\
\hline 10 & RING & 9 & 8 & 7 & 6 & 5 & 4 & 3 & 2 & 1 & 2 & 3 & 4 & 5 & 6 & 7 & 8 & 9 & TARGET \\
\hline 11 & RING & 9 & 8 & 7 & 6 & 5 & 4 & 3 & 2 & 1 & 2 & 3 & 4 & 5 & 6 & 7 & 8 & 9 & CONTROLS \\
\hline 12 & RING & 9 & 8 & 7 & 6 & 5 & 4 & 3 & 2 & 1 & 2 & 3 & 4 & 5 & 6 & 7 & 8 & 9 & CONV FAC \\
\hline 13 & TARGET & 9 & 8 & 7 & 6 & 5 & 4 & 3 & 2 & 1 & 2 & 3 & 4 & 5 & 6 & 7 & 8 & 9 & CONTROLS \\
\hline 14 & TARGET & 9 & 8 & 7 & 6 & 5 & 4 & 3 & 2 & 1 & 2 & 3 & 4 & 5 & 6 & 7 & 8 & 9 & CONV FAC \\
\hline 15 & CONTROLS & 9 & 8 & 7 & 6 & 5 & 4 & 3 & 2 & 1 & 2 & 3 & 4 & 5 & 6 & 7 & 8 & 9 & CONV FAC \\
\hline
\end{tabular}

1=Equal $3=$ Moderate $5=$ Strong $7=$ Very Strong $9=$ Extreme

Each expert was initially asked to draw a chart (such as that shown below in Exhibit 3) representing his opinion of the relative rankings of each subproject relative to the comple xity parameter.

Exhibit 3. Rank order decision aid used during expert elicitation.

\begin{tabular}{|llll|}
\hline & & COMPLEXITY & \\
Score & & & \\
& & & \\
\hline & Controls & Ring, & Source, \\
& & Linac & Conventional facilities, \\
& & & Target \\
& $(\mathrm{x} 3)$ & $(\mathrm{x} 3)$ & \\
& & & \\
& & & \\
& &
\end{tabular}


In this example, the expert ranked the controls system the most complex; the ring and linac systems equal in complexity; and the source, conventional facilities, and target systems equal and least complex. The experts provided not only this relative ranking, but also an initial estimate of separation between subprojects. Thus the chart provided a first cut which was later refined in greater detail. The chart device greatly alleviated confusion and frustration experts faced in completing the overwhelming number of paired comparisons in the Expert Choice ${ }^{\mathrm{TM}}$ graphical interface.

The above procedure was repeated for design immaturity and stressful environment for reliability, and for detection/diagnosis time, preparation time, correction time, and start-up time for maintainability. The end result is a priority ranking for reliability and maintainability for each expert. Exhibit 4 shows the reliability priority rankings for each subproject for the example SNS expert. There is a similar representation of maintainability priority rankings. These priority rankings, substituted into Equations 7 and 8 give the following overall facility $90 \%$ availability apportioned among subprojects.

Exhibit 4. Single-expert priority rankings computed by Expert Choice ${ }^{\mathrm{TM}}$.

ORNL DWG 99C-161

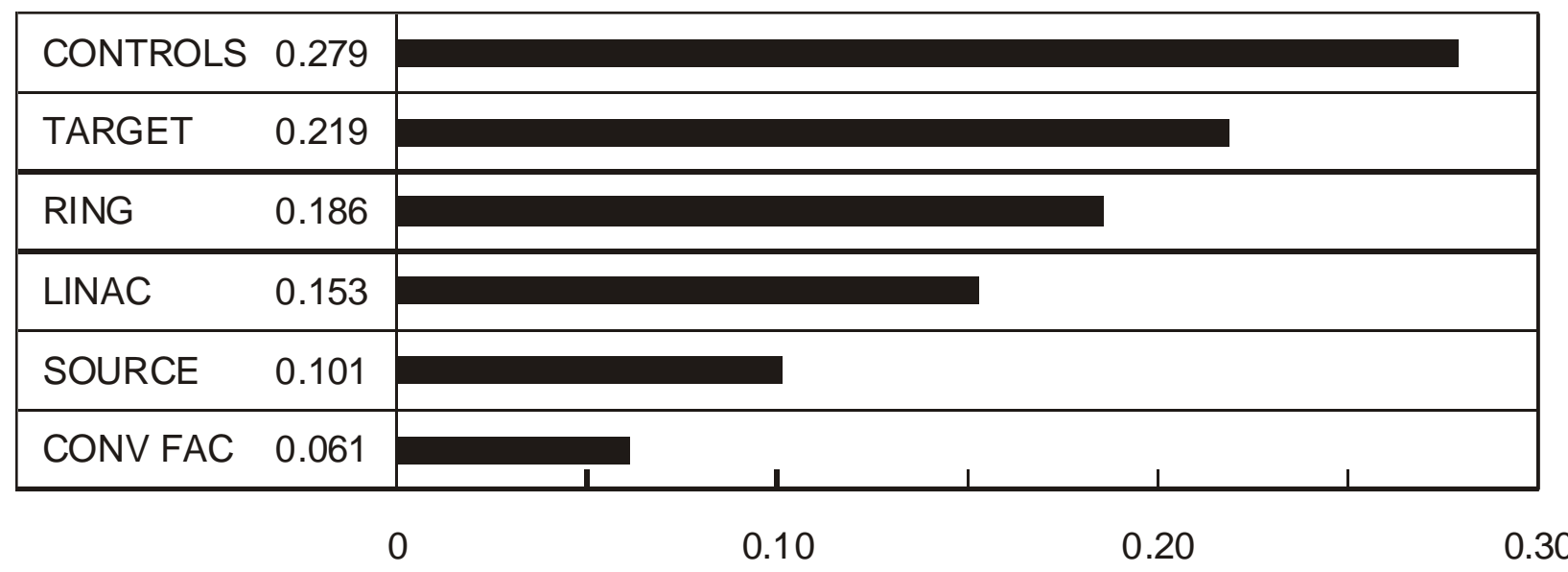

\subsection{EXPERT AGGREGATION FOR THE SNS}

For this example, a structured interview was conducted individually for each expert. The aggregation technique used in the SNS Project was to weight each expert judgement equally. Thus, biases should be evenly distributed. Table 1 shows the final aggregate priority rankings for each subproject for the example application to the SNS facility. The control systems, conventional facilities and source were allocated extremely high availability scores, whereas, the target systems were awarded the lowest aggregate availability requirement. 
Table 1. SNS availability allocations.

\begin{tabular}{llcc}
\hline W.B.S. & Subsystem & Availability (\%) & Downtime (per 6 wks.) \\
\hline 1.3 & Front End (source) & 99.5 & 05.0 hours \\
1.4 & LINAC & 97.5 & 25.6 \\
1.5 & Ring \& Transfer Line & 96.9 & 32.0 \\
1.6 & Target & 96.3 & 38.4 \\
1.7 & Experiment Systems & 100.0 & 00.0 \\
1.8 & Conventional Facilities & 99.7 & 03.0 \\
1.9 & Control Systems & 99.7 & 03.0 \\
\hline
\end{tabular}

The covariance structure was examined to evaluate the degree of agreement among experts with respect to the availability priority rankings. The correlation matrix in Table 2 demonstrates a consistent, high reliability in priority rankings of sub-programs. The range of correlations among expert pairs was from 0.685 to 0.985 , with $73 \%$ of the correlations above 0.800 . The correlations of expert's priority rankings with the aggregate scores ranged from 0.742 to 0.988 . Apparently, the aggregate scores represented each individual expert's responses very well.

Table 2. Correlation matrix of subsystem rankings for experts and facilities

\begin{tabular}{lllllllllll}
\hline & TG & JT & RW & BD & RY & DS & Agg & ISIS & AGS & LANCE \\
\hline TG & 1.0 & .938 & .943 & .770 & .733 & .778 & .968 & .197 & .940 & -.426 \\
JT & .938 & 1.0 & .933 & .813 & .685 & .866 & .988 & .399 & .885 & -.158 \\
RW & .943 & .933 & 1.0 & .853 & .823 & .848 & .974 & .275 & .972 & -.344 \\
BD & .770 & .813 & .853 & 1.0 & .872 & .985 & .841 & .695 & .971 & -.297 \\
RY & .733 & .685 & .823 & .872 & 1.0 & .806 & .742 & .404 & .759 & -.230 \\
DS & .778 & .866 & .848 & .985 & .806 & 1.0 & .869 & .740 & .959 & -.197 \\
Agg & .968 & .988 & .974 & .841 & .742 & .869 & 1.0 & .345 & .960 & -.292 \\
ISIS & .197 & .399 & .275 & .695 & .404 & .740 & .345 & 1.0 & .873 & .285 \\
AGS & .940 & .885 & .972 & .971 & .759 & .959 & .960 & .873 & 1.0 & -.443 \\
LANCE & -.426 & -.158 & -.344 & -.297 & -.230 & -.197 & -.292 & .285 & -.443 & 1.0 \\
\hline
\end{tabular}

The agreement of the aggregate scores with availability data from other accelerator facilities was widely variable, reinforcing the conclusion that previous data from similar facilities cannot blindly be used to apportion availability for a one-of-a-kind facility. The AGS availability scores agreed extremely well with the aggregate scores for the SNS (.96); the agreement with ISIS was moderate (.345); and, the correlation with LANSCE H+ was actually negative (-.292).

The availability scores for each expert were submitted to a principal components analysis in order to represent expert judgment in a lower dimensional space. The expert's scores are plotted with respect to the first two principal components in Figure 1. Three natural clusters emerged. The largest cluster consisted of the experts representing the ring, target, and conventional facilities. The aggregate scores were also located in this cluster. A smaller cluster contained the experts for the Linac and source, and a one-member cluster represented the source. These two smaller clusters differed from the main cluster 
mainly in their estimates of higher relative target availability. The singleton outlier cluster also differed from other experts by allocating a lower control systems availability.

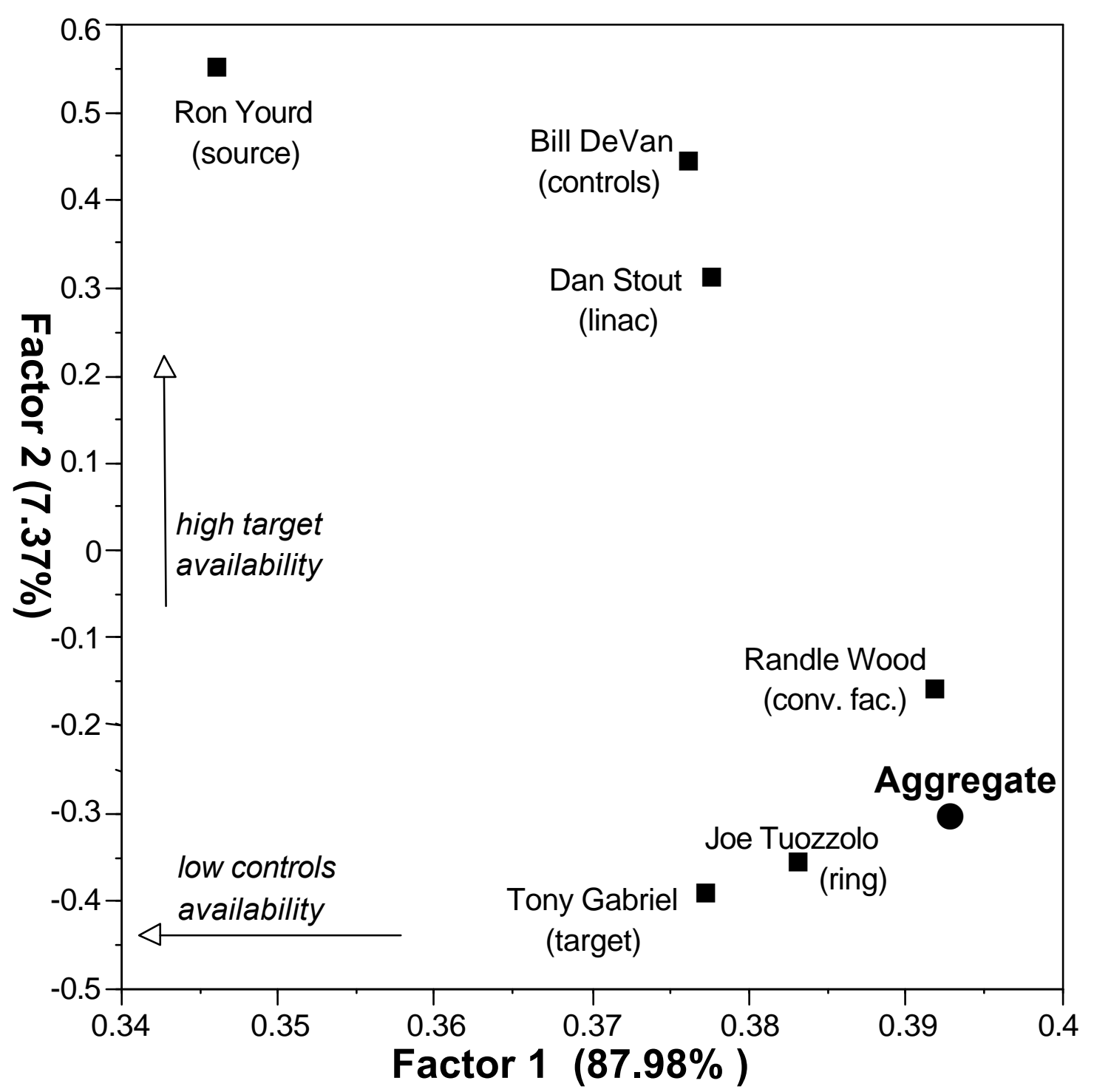

Figure 1. Principal component scores show agreement between expert judgments and aggregate allocations.

\section{CONCLUSIONS}

Systems design engineers must translate overall facility requirements into detailed design and operating specifications for the numerous systems and subsystems that make-up a facility. This work presents a top-down method that distributes overall facility availability requirements among facility systems based on 
each system's capability for meeting a design objective. Availability apportionment should be considered initially during early conceptual design and at the conclusion of each design phase or redesign.

A mathematical model is derived that relates the apportioned availability of the $i^{\text {th }}$ system, $A_{i}$, to the overall facility availability goal, A, and failure rate and repair rate weighted rankings, $\mathrm{z}$ and $\mathrm{y}_{\mathrm{I}}$, respectively. Weighted rankings are determined from quantitative analysis of expert judgments. The choice of methods for expert selection, bias, elicitation and aggregation are important. In the example application of the method to the SNS Project, the experts were subproject senior team leader (or designated person) and senior project management. Thus, each technical area was equally represented and biases should be evenly distributed. Judgements were elicited from each expert individually and each expert opinion was weighted equally. Team aggregation and final adjustments of availability allocations were performed at the conclusion of the structured interviews, and results were reviewed.

Suggested failure rate criteria are system complexity, design immaturity, operating time, and stressful environment. Repair rate criteria are fault detection time, fault diagnosis time, preparation time, fault correction time, and checkout time. The selection of criteria should be tailored to each application. A paired comparison method is recommended for eliciting priority rankings. The Analytic Hierarchy Process (AHP) is used to produce a set of priority rankings for each alternative. The criteria were ranked initially for the SNS example. Experts were asked, for example, to compare complexity and design maturity with respect to their importance in apportioning reliability. The priority rankings of criteria are determined using an eigenvalue method. After completing the ranking of criteria, each alternative subproject (e.g., source, linac, ring, target in the example SNS application) pair is evaluated with respect to each criterion. This process is repeated for maintainability criteria and alternatives. After completing a matrix of paired comparisons, the expert is allowed to view the resultant priority ranking and consistency index. If the ranking seems counterintuitive, or the consistency index too high, the expert is allowed to revise their judgements. The Expert Choice ${ }^{\mathrm{TM}}$ commercial software was used on a laptop computer to assist the expert in working thorough the AHP method. Once the results were synthesized, the weighting factors were input to the derived mathematical model and apportioned systems availability calculated.

Apportionment of overall availability among systems and subsystems is beneficial because it quantifies design requirements rather than using the "we will do the best that we can" approach regarding design for reliability and maintainability. Comparisons of system availability design requirements and predicted availability during the design process reveal weak links or critical components that prevent achieving the availability requirement. Resolving these weak links improves the state-of-the-art of the design. The apportionment process enhances design integration by focusing attention on the relationship between facility, system, subsystem, and component availability. Requirements determined through an apportionment process will be more realistic and attainable than those resulting from retrofits in the field.

This top-down apportionment method results in optimum facility availability since the method imposes higher availability requirements on those systems in which an incremental increase in availability is easier to achieve, and lower availability requirements where an increase is more costly. Availability is allocated based on a joint consideration of reliability and maintainability. 


\section{REFERENCES}

1. Kececiogly, Dimitri, (1991). Reliability Engineering Handbook, Vol 2, Chapter 15, Prentice Hall, ISBN 0-13-772302-4.

2. Advisory Group of Reliability of Electronic Equipment (AGREE), Reliability of Military Electronic Equipment, Office of the Assistant Secretary of Defense Research and Engineering, Washington, D.C. June 4, 1957.

3. Karmiol, E.D., Reliability Apportionment, Preliminary Report EIAM-5, Task II, General Electric, Schenectady, N.Y., pp. 10-22, April 8, 1965.

4. Smedley, K. "Reliability analysis for LEB ring magnet power system in SSC," IEEE Transactions on Nuclear Science, 39(4), pp. 1170-1174, 1992.

5. Bracha, V.J., “The Methods of Reliability Engineering,” Machine Design, pp. 70-76, July 30, 1964.

6. Haire, M.J., J. G. Maltese, and R.G. Sohmer, “A System Availability ‘Top-Down' Apportionment Method," 1985 Proceedings of the Annual Reliability and Maintainability Symposium, Institute of Electrical, and Electronic Engineers.

7. Virtanen, S., "Reliability in Product Design - Specification of Dependability Parameters," 1998. Proceedings of the Annual Reliability and Maintainability Symposium, Institute of Electrical, and Electronic Engineers.

8. Saaty, T.L. Fundamentals of Decision Making and Priority Theory with the Analytical Hierarchy Process (Vol. 6), Pittsburgh, PA: RWS Publications, 1994.

9. Saaty, T.L. The Analytic Hierarchy Process, New York: McGraw Hill Co., 1980.

10. Aggarwal, K.K., and Y. Singh, "Software reliability apportionment using the analytic hierarchy process", SIGSOFT Software Engineering Note, Vol. 20, No. 5, pp. 56-61, 1995.

11. Alexander, J.M. "Priorities and Preferences in Conflict Resolution", Mathematics and Computers in Simulation 25/2, 108-119. 1983.

12. Algie, J., G. Mallen and W. Foster, "Financial Cutback Decisions by Priority Scaling", Journal of Management Studies 20/2, 233-260, 1983.

13. Arbel, A. and A. Seidmann, "Performance Evaluation of Flexible Manufacturing Systems", IEEE Transactions on Systems, Man and Cybernetics SMC/14/4, 606-617, 1984.

14. Cambron, K.E. and G.W. Evans, "Layout Design Using the Analytical Hierarchy Process", Computers \& IE 20/2, 221-229, 1991. 
15. Dolan, J.G. "Can Decision Analysis Adequately Represent Clinical Problems?", Journal of Clinical Epidemiology 43/3, 277-284, 1990.

16. Hamalainen, R.P. and M. Poyhonen, "On-line group decision support by preference programming in traffic planning", Group Decision and Negotiation, Vol. 5, pp. 485 - 500, 1996.

17. Hamalainen, R.P., K. Oehlandt, T., Seppalainen and J. Ruusunen,"An approach to decision support in national energy policy planning", Proceedings of the IEEE Conference on Systems, Man and Cybernetics, Tucson pp.1008-1012, 1985.

18. Hussien, F.M., M.A. Obeid and K.S. El-Malahy, "Site Selection of a Dual Purpose Nuclear Power Plant in Saudi Arabia", Nuclear Technology 79/3, 311-321, 1987.

19. Lyon, Tim D., Farrokh Mistree, "A computer-based method for the preliminary design of ships", Journal of Ship Research, Vol. 29, No. 4, December, 251-269, 1985.

20. Saaty, T.L., "Risk - Its Priority and Probability: The Analytic Hierarchy Process", Risk Analysis 7/2, 159-172, 1987.

21. Saaty, T.L. and L.G. Vargas, "Implementing Neural Firing: Towards a New Technology", Mathl. Comput. Modelling, Vol. 26, No. 4, pp. 113-124, 1997.

22. van Steen, J.F.J., "A perspective on structured expert judgment”, Journal of Hazardous Materials, 29, 365-385, 1992.

23. Zio, E., "On the use of the analytic hierarchy process in the aggregation of expert judgments", Reliability Engineering and System Safety, Vol. 53, 127-138, 1996. 


\title{
INTERNAL DISTRIBUTION
}

\author{
1. J. Barhen \\ 2. J. M. Begovich \\ 3. J. E. Cleeves \\ 4-9. M. J. Haire \\ 10. R. L. Kustom \\ 11. T. L. Mann Jr. \\ 12. S. C. Nelson Jr. \\ 13. K. Reece \\ 14-19. J. C. Schryver \\ 20. L. E. Temple Jr \\ 21. D. R. Tufano \\ 22. T. Zacharia \\ 23. Central Research Library \\ 24. ORNL Laboratory Records-RC \\ 25-26. ORNL Laboratory
}

None. 